
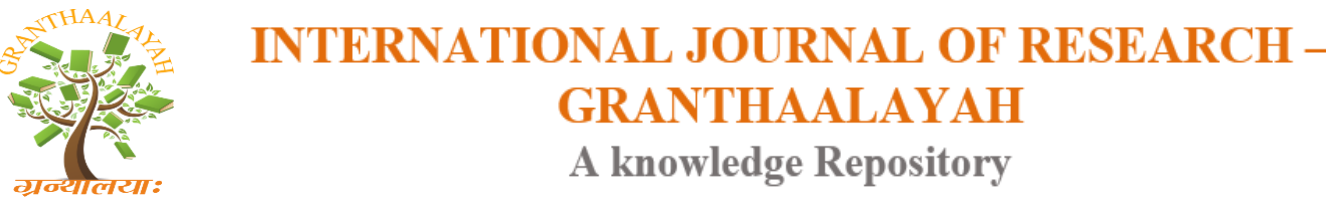

Science

\title{
RELATIVE IMPACTS OF DIFFERENT DIETS ON THE QUALITIES OF ERI SILK COCOON AND ERI SILK YARN OF FOUR DIFFERENT MUTANTS STRAINS OF PHILOSAMIA RICINI Bsd.
}

\author{
Akhilesh Kumar Gahlaut ${ }^{* 1}$, Dr. J. Arjun ${ }^{2}$ \\ ${ }^{* 1}$ P.G. Department of Zoology, Magadh University, Bodh-Gaya, INDIA \\ ${ }^{2}$ Department of Zoology, Lumding Collage, Lumding 782447, Assam, INDIA
}

DOI: https://doi.org/10.29121/granthaalayah.v4.i11.2016.2422

\section{ABSTRACT}

The relative impact of different food plants (diets) namely foliages of castor, kesseru, payam and Topioca plants on the four different mutant strain viz; yellow plain, yellow zebra,G.B Plain ,G.B Zebra in respect of qualities of their eri cocoon and eri silk yarn have been evaluated under the reeling parameters. Result indicate that among the four different food plants like foliages of castor followed by kesseru have been found evidently better than the foliages of payam and Topiocal in respect of average cocoon weight (gm)4.10, 3.55, 3.98, 3.10, Average shell weight (gm) 0.55, 0.45, 0.52, 0.41, Average shell ratio 13.91, 12.0, 13.0, 11.55, Average lengt- $h$ of silk yarn (mtrs) 2510, 2350, 2498, 2285, Average size of eri silk (D)13D, 11D, 12D, 19D, Average production of eri silk per hr(gm)13.70, 12.50, 13.10, 11.95, Average tenacity of fibre $(\mathrm{g} / \mathrm{d})$ 14.0, 11.0, 12.0, 10.0. As far as qualitative differences of all the four food plants in relation to qualities of eri cocoon and eri silk yarn are concerned the castor along with kesseru food plants have achieved the status of supremacy primary food plant and payam and tapioca the states of secondary food plants.These variation show that the qualities and production of eri silk cocoon and eri silk yarn depends on the different food plants.

Keywords:

Philosamia ricini Bsd. Castor,Kesseru,Payam, Topioca, Variation and Qualities.

Cite This Article: Akhilesh Kumar Gahlaut, and Dr. J. Arjun, "RELATIVE IMPACTS OF DIFFERENT DIETS ON THE QUALITIES OF ERI SILK COCOON AND ERI SILK YARN OF FOUR DIFFERENT MUTANTS STRAINS OF PHILOSAMIA RICINI Bsd." International Journal of Research - Granthaalayah, Vol. 4, No. 11 (2016): 77-81.

\section{INTRODUCTION}

The rearing of silk producing insects which are numerous in nature constitutes a branch of science known as sericulture. India has the unique distinction of being the only country in the world which produces all the four popular varieties of natural silks viz; mulberry, tasar, eri and munga. Among the non-mulberry silkworm, the Philosamia ricini Bsd is the main eri silkworm 
commonly reared on different host plants in Assam, Kerala, West Bengal and some part of Bihar and Jharkhand. The adult mouth is stout and dark and the wings are darkish brown and white. It deposits 120-200 eggs in cluster. The caterpillars feed on the foliages of castor and kesseru which is primary food plants. Larval period varies between 17 to 25 days. The four different mutant strains of Philosamia ricini viz; yellow plain, yellow zebra, G.B Plain, G.B Zebra has been evaluated under the reeling parameters in different seasons like May-June, July-August, September-October and November-December. Among four mutant strains Yellow plain is the best in all respect of productivity in month of May to June.

\section{MATERIALS AND MATHODS}

The eri cocoon were collected from Ranchi (Jharkhand), the seed supply station in the month of June 2013 and carefully transported to Bodh-Gaya and acclimatized under the laboratory condition. The healthy cocoons of Philosamia ricini of four different strains namely yellow plain, yellow zebra, G.B Plain and G.B Zebra with uniform weight and size were selected for the present experiment. The male and female moths were put in bigger bamboo baskets for pairing .The diseases free eggs of different strains of Philosamia ricini were washed with 5\% formalin solution for 5 minutes for surface sterilization. The disinfected eggs of different strain were kept in specially designed egg boxes having transparent top to permit light and perforated side for aretion.

\section{RESULT AND DISCUSSION}

The relative impacts of different food plants (diets) namely foliages of castor, kesseru, payam and tapioca plants on the four different mutant strains viz; Yellow plain, Yellow zebra, G.B. plain and G.B. zebra in respect of qualities of their eri cocoons and eri silk yarn have been evaluated and results obtained after the relative analysis have been recorded in following, It reveals the relative impacts of different food plants namely castor, kesseru, payam and topioca on the cocoon weight $(4.10,3.92,2.75$ and $2.45 \mathrm{gms}$.), shell weight $(0.55,0.52,0.41$ and $0.37 \mathrm{gms}$.$) , shell ratio (13.91,12.83,11.98$ and $11.65 \%)$, filament length $(2510,2490,2312$ and $2300 \mathrm{mtrs}$.), size of eri silk (13D, 12D, 9D and 8D), rate of production of eri silk $(13.70,13.62$, 12.15 and $12.10 \mathrm{gms}$.$) and tenacity of fibre (1.98,1.72,1.53$ and $1.42 \%)$ on the qualities of eri cocoons and eri silk yarn of Yellow plain mutant strain of Philosamia ricini. The results obtained are indicative of under given facts: Different diets of eri silk worm namely foliages of castor, kesseru, payam and tapioca evidently influence the qualities of eri cocoon and eri silk yarn showing significant impacts of different diets on the reeling performances of Yellow plain mutant strain of Philosamia ricini in respect of qualities of eri cocoons and eri silk fabrics. Among the four different food plants the relative impacts of foliages of castor followed by kesseru have been found evidently better than the foliages of payam and tapioca in respect of the qualities of eri cocoons and eri silk yarn of yellow plain mutant strain. It reveals the relative impacts of four different food plants on the qualities of cocoon weight, shell weight, shell ratio, length of fibre, size of fibre, production rate of eri silk fibre, tenacity of eri silk and elongation percentage of silk fibre of Yellow zebra mutant strain of Philosamia ricini. It clearly indicates that the cocoon weight $(3.55,3.40,2.30$ and $2.21 \mathrm{gms}$.), shell weight $(0.45,0.42,0.31$ and 0.28 gms.), shell ratio (12.10, 11.65, 10.93 and 10.12\%), length of eri silk fibre $(2350,2340,2290$ and 2270mtrs.), size of eri silk (11D, 10D, 9D and 8D), production rate of silk fibre (12.50, 
$12.10,11.35$ and $11.02 \mathrm{gms}$.$) , tenacity of fibre (1.81,1.70,1.20$ and $1.10 \mathrm{~g} / \mathrm{d})$ and elongation of eri silk fibre $(11.0,10.0,9.0$ and 8.0) in relation to culture of yellow zebra mutant strain on the foliages of castor, kesseru, payam and tapioca food plants respectively present significant variations in respect of qualities of eri cocoons and eri silk yarn. impact of different food plants (diets) on the qualities of silk cocoon and yarn like other mutant strain. It is further clear that the qualities of eri silk cocoon along with eri silk yarn are relatively better on castor food plant followed by kesseru food palnt than payam and tapioca food palnts. It appears that castor and kesseru food plants have registered their supermacy as primary food plants over payam and tapioca secondary food plants as far as dietary impacts of foliages of four different plants on the qualities of reeling performances in respect of qualities of eri cocoon and eri yarn of G.B. mutant strain of Philosamia ricini are concerned.

Lastly the relative impacts of four different food plants (diets) on the qualities of eri silk cocoon and eri silk yarn of G.B. zebra mutant strain of Philosamia ricini have been evaluated and results obtained have been recorded in following is clearly indicates that the qualities of eri silk cocoon and eri yarn in respect of cocoon weight (3.10, 2.95, 2.20 and 2.10gms.), shell weight (0.41, $0.40,0.31$ and $0.29 \mathrm{gms}$.), shell ratio $(11.55,11.41,10.0$ and $9.95 \%)$, length of silk fibre (2285, 2270, 2210 and 2200mtrs.), size of eri silk (9D, 8D, 7D and 6D), production of eri silk per hour $(11.95,11.73,11.04$ and $11.02 \mathrm{gms}$.), tenacity of fibre $(1.32,1.25,1.04$ and $1.04 \mathrm{~g} / \mathrm{d})$ and elongation of eri silk fibre (10.0, 9.0, 8.0 and 7.0\%) respectively in relation to castor, kesseru, payam and tapioca food plants present variations in respect of qualities of eri cocoons and eri silk yarn of G.B. zebra mutant strain of Philosamia ricini showing thereby significant impacts of dietary variations on the reeling performances of G.B. zebra mutant strain of Philosamia ricini. Finally the relative impacts of all the four different types of food plants (castor, kesseru, payam and tapioca) on the qualities of eri cocoons and eri silk yarn of four mutant strains (Yellow plain, Yellow zebra, G.B. plain and G.B. zebra) of Philosamia ricini. Which accounts for the under given facts: All the four different food on account of their relative nutritional difference present significant variations on the qualities of eri cocoons and eri silk yarn of four mutant strains of Philosamia ricini? The qualities of eri cocoon and eri silk yarn of all the mutant strains in spite of relative differences are by and large relatively better on castor and kesseru food plants than the payam and tapioca food plants. As far as qualitative differences of all the four food plant in relation to qualities of eri cocoon and eri silk yarn are concerned the castor along with kesseru food plants have achieved the status of primary food plants and payam and topioca the states of secondary food plants. 


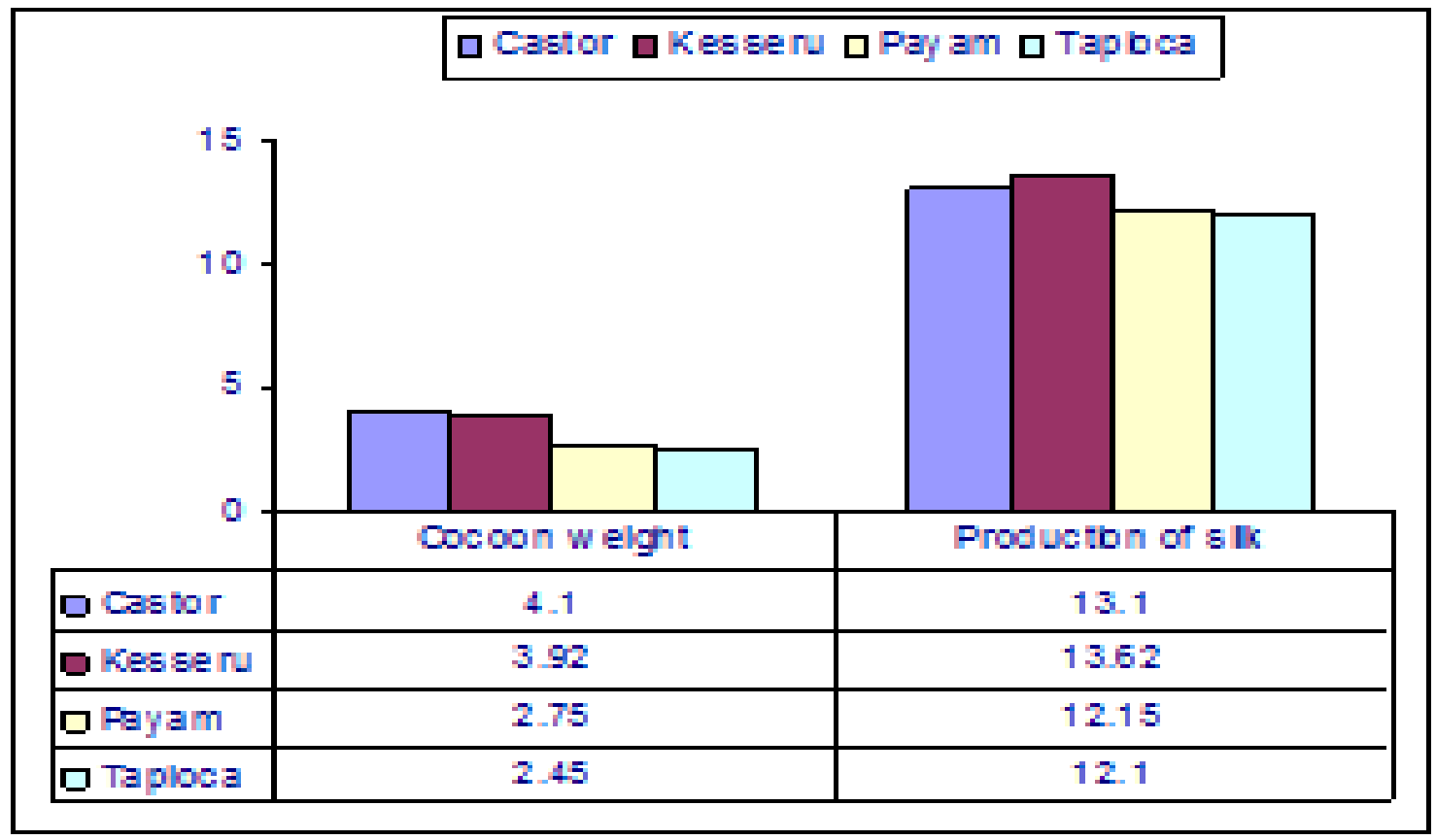

Figure 1: Showing relative impacts of four different food plants on the cocoon weight and rate of production of eri silk of yellow plain mutant strain of Philosamia ricini Bsd.

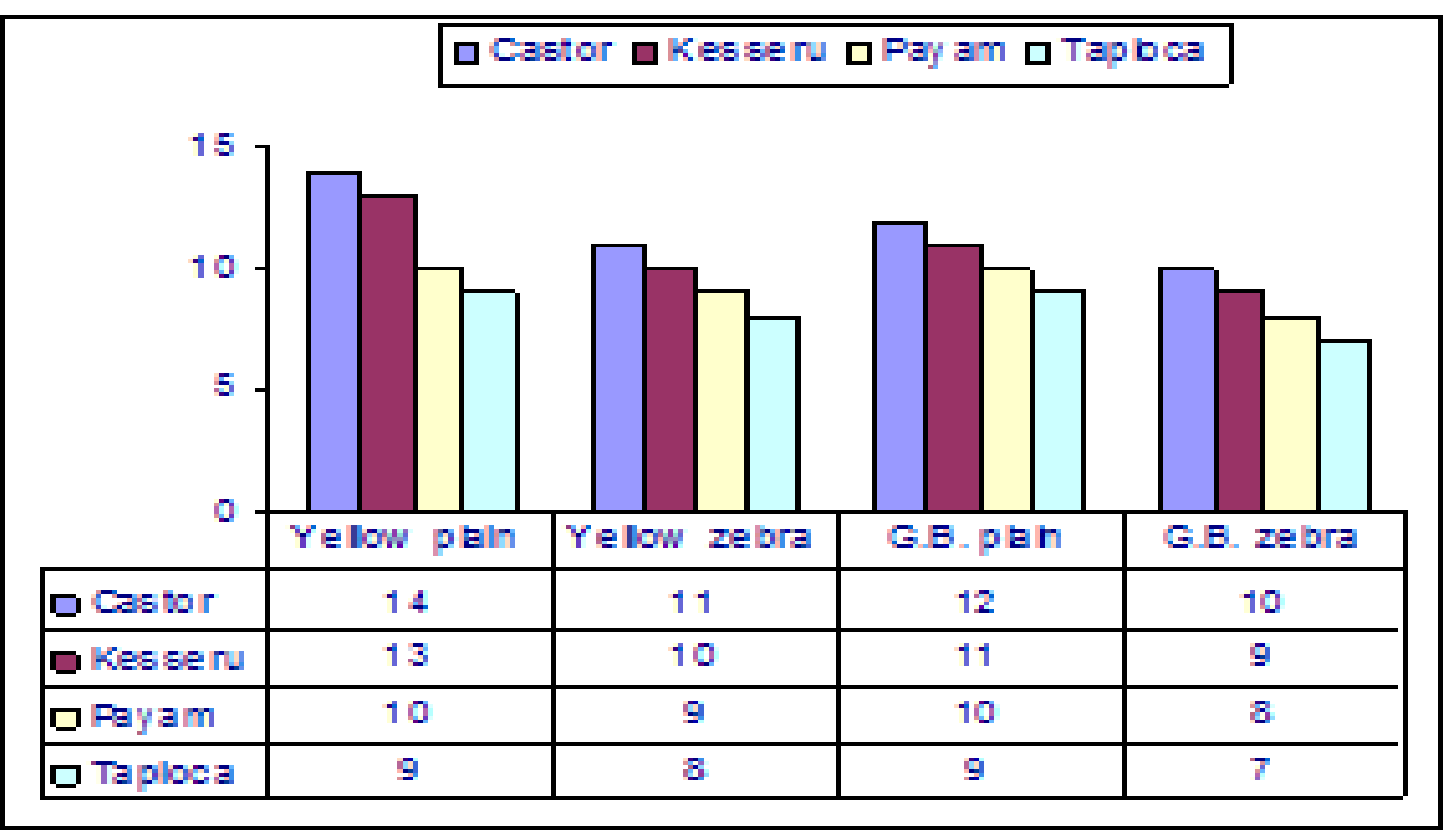

Figure 1: Showing relative impacts of four different food plants (diets) on the size of eri silk yarn of four different strain of Philosamia ricini Bsd. 


\section{CONCLUSION}

In various states such as Assam, Bihar, Bangal, Jharkhand where Eri silkworm are usually reared on different food plants .It produces brick red and white erisilk, The fabric made from this silk is held in high esteem. Finally the relative impacts all the four different types of food plant (Castor, Kesseru, Payam and Topioca) on the qualities of eri cocoon and erisilk yarn of four mutant strains (Yellow plain, Yellow zebra, G.B plain and G.B zebra) of philosamia ricini have been found castor and kesseru food plants are relatively better nutritional value.

\section{REFERENCES}

[1] Singh,B.K and Das,P.K (2003)Eco-races of eri silkworm indian silk (5);7-10.

[2] Das.B.Mahanta,J.C and Jaiprakash,P. Singh,B.K.(2012) Bio-resources of eri silkworm and its host plants of north east India,Utilization and need for conservation,eco-scane $\operatorname{vol}(1)$ sp.473-478.

[3] Siddique,A.A.Agricultural activvity and castor plantation for eri silk production India silk (6) 37-4 (2009).

[4] Gomma,A Ahmad.Biological studu on the eri silkworm,Attacus ricini bosid.Indian j.Seric (1);81-88.(1972).

[5] Sharma,K.B AND KUMAR,P.(1997);Effects of ecological factors on the biology of Indian Tasar silkworm,Antheraea Mylitta D (Saturniidae;Lepidoptera);Recant advances in Ecological research 215-223.

[6] Roy,P. and Das,P. (2010) Performance of promosing eco races of Eri silkworm (philosamia ricini) in agro climatic condition of west odisha.The Bio-scane 51(12);201205.

[7] Jolly,,M.S.(1971);A new technique of rearing silkworm.Indian silk,Feb.17-18.

[8] Kumar,R.(2010);Rearing performances eri silkworm in mansoon season in U.P.Asian j. Biol.Sei.(12)303-310.

[9] Donnel (2010); The wonder of wild silk,spinnoff.24(1);53-55.

[10] Akai,Hiromu (1998)Global Scenario of wild silk,Indian silk (37)687;18-20.

[11] Kar,J.(2009);Survey on Eri researches in orissa.Directorate of Textile.pp.45-48. 